\title{
Epigenome-wide association in adipose tissue from the METSIM cohort
}

\author{
Luz D. Orozco ${ }^{1, *, \dagger}$, Colin Farrell ${ }^{1}$, Christopher Hale ${ }^{1}$, Liudmilla Rubbi ${ }^{1}$, \\ Arturo Rinaldi ${ }^{1}$, Mete Civelek $^{2, \ddagger}$, Calvin Pan ${ }^{2}$, Larry Lam $^{1}$, Dennis Montoya ${ }^{1}$, \\ Chantle Edillor ${ }^{2}$, Marcus Seldin ${ }^{2}$, Michael Boehnke ${ }^{3}$, Karen L. Mohlke ${ }^{4}$, \\ Steve Jacobsen ${ }^{1,5,6}$, Johanna Kuusisto ${ }^{7}$, Markku Laakso ${ }^{7}$, Aldons J. Lusis ${ }^{2}$ and \\ Matteo Pellegrini ${ }^{1,5}$
}

${ }^{1}$ Department of Molecular, Cell and Developmental Biology, University of California Los Angeles, ${ }^{2}$ Departments of Human Genetics, Medicine, and Microbiology, David Geffen School of Medicine, University of California Los Angeles, Los Angeles, CA 90095, USA, ${ }^{3}$ Department of Biostatistics and Center for Statistical Genetics, University of Michigan, Ann Arbor, MI 48109, USA, ${ }^{4}$ Department of Genetics, University of North Carolina, Chapel Hill, NC 27599, USA, ${ }^{5}$ Eli \& Edythe Broad Center of Regenerative Medicine \& Stem Cell Research, ${ }^{6}$ Howard Hughes Medical Institute, University of California Los Angeles, Los Angeles, CA 90095, USA and ${ }^{7}$ Institute of Clinical Medicine, Internal Medicine, University of Eastern Finland and Kuopio University Hospital, Puijonlaaksontie 2, 70210 Kuopio, Finland

*To whom correspondence should be addressed. Tel: +1 3234914999; Fax: (310) 206-3987; Email: luz.d.orozco@gmail.com

Human Molecular Genetics, 2018, 27(10), 1830-1846.

doi: $10.1093 / \mathrm{hmg} /$ ddy093

Five figures were erroneously omitted from the online supplemental material upon publication. The supplemental material section has now been updated to include these figures.

The publisher regrets the error.

${ }^{\dagger}$ Present address: Department of Bioinformatics, Genentech, Inc., South San Francisco, CA 94158, USA.

${ }^{\ddagger}$ Present address: Department of Biomedical Engineering, Center for Public Health Genomics, University of Virginia, Charlottesville, VA 22904, USA.

(c) The Author(s) 2018. Published by Oxford University Press. All rights reserved.

For permissions, please email: journals.permissions@oup.com 